\title{
Belonging to Tomorrow: An Overview of Procrastination
}

\author{
Brian A. Wilson \\ Peacock Foundation \\ PO Box 21154, Glendale, Ca 91221, USA \\ Tuyen D. Nguyen (Corresponding author) \\ California State University \\ PO Box 6868, Fullerton, Ca 92834, USA \\ E-mail: Trentspringfield@yahoo.com
}

Received: January 6, 2012

Accepted: January 10, 2012 Published: March 1, 2012

doi:10.5539/ijps.v4n1p211

URL: http://dx.doi.org/10.5539/ijps.v4n1p211

\begin{abstract}
Available material indicates that the formal study of procrastination is a relatively recent occurrence. This literature review examines the work of some of the most prominent researchers in this field. This paper will examine the underlying causes of procrastination as delineated by the most current research. In addition, it will explore the hypothesis that procrastination exists on a scale, and that in its most elevated state can have severe, substantial and lasting consequences. Finally, this paper will review some methods for blunting the impact of procrastination, and briefly examine what lies ahead in the study of this condition.
\end{abstract}

Keywords: Procrastination, Irrational delay, Pathological decision-making, Trait procrastination, Chronic delay, Avoidant procrastination, Arousal procrastination

\section{Introduction}

In "Measure for Measure," William Shakespeare wrote, "Our doubts are traitors, and make us lose the good we oft might win by fearing to attempt" (Shakespeare, 1997). Though he may have addressed the issue of procrastination most poetically, he was by no means the first to do so. Indeed, Knaus (2000) calls procrastination an "ancient nemesis," saying that it parallels human civilization and may have originated 2.5 million years ago. Prescriptions against the undermining influence of this inaction can be found in the sage codes of Babylonian leader Hammurabi (Knaus, 2000) Roman emperor Marcus Aurelius (121-180 A.D.) cautioned against unnecessary delays (Aurelius, 1864, as cited in Knaus, 2000). More recently, Benjamin Franklin included "performing without fail what you resolve" as one of his 13 virtues (Franklin, 1989, as cited in Knaus, 2000), thus speaking out against the evils of procrastination. The origin of the word procrastination itself derives from the late 16th century Latin procrastinat- "deferred until tomorrow", from the verb procrastinare, from pro"forward" plus crastinus "belonging to tomorrow" (Apple, 2005).

Despite its long history, the subject of procrastination received little attention in the United States prior to the late 1970s (Knaus, 2000). And it was not until 2007 that Steel produced a meta-analytic and theoretical review of the nature of the problem, by then having some 621 sources to comb for information. Even today, research on the matter seems fractured. Studies reach competing conclusions, or differing conclusions are drawn from the same data. Still, some clear findings have emerged, many subverting popular beliefs about the causes and treatments of this problem.

But is procrastination even a problem? Or as Andreou (2007) muses, is it properly tempting to suppose that, as the rational choice theory advances, preferences are revealed in choice and therefore there are no cases of procrastination? Does it interfere with occupational, academic or personal situations? Does it deserve a place in the DSM-V or DSM-VI? Or is it a sporadic, common but ultimately harmless behavior to be laughed off, and laughed at? After all, virtually all of us - young, old, of any and all races, male or female--will, sheepishly, admit 
to having procrastinated over a matter large or small, and probably having done so on numerous occasions. For example, 80-95\% of college students engage in procrastination (Ellis \& Knaus, 1977; O’Brien, 2002, as cited in Steel, 2007). Procrastination does seem to go hand in hand with the human condition, across cultures (Ferrari, O'Callaghan and Newbegin, 2005) and throughout time (Knaus, 2000; Kotler, 2009; Achor, 2010).

The topic of procrastination was one of nearly a dozen considered as a possible focus for this paper. These other possibilities were considered and researched, but ultimately discarded as procrastination kept rising to the top of the stack. It held the most resonance with the author, as procrastination has been an issue, to a greater and lesser degree, throughout his life. Most recently, procrastination was a major, contributing factor to his decision to change careers from Hollywood writer to the world of psychology and therapy. Aversion to unpleasant tasks, discounting of distant goals, giving in to immediate gratification - all the aspects of procrastination delineated in this paper - combined to contribute to a diminished level of success in Hollywood. On the one hand, the author finds it comforting and normalizing to see that research has addressed this very condition; on the other, it is disappointing to realize that the research is so relatively new and still somewhat disorganized that it did not appear to be readily available to therapists seen by the author, and so solutions could not be brought to bear while the problem was happening.

Through review of existing literature, this paper will attempt to close that knowledge gap for the reader by examining the underlying causes of procrastination as delineated by the most current research. In addition, it will explore the hypothesis that procrastination exists on a scale, and that in its most elevated state can have severe, substantial and lasting consequences. Finally, this paper will review some methods for blunting the impact of procrastination, and briefly examine what lies ahead in the study of this condition.

\section{Methods and Results}

Research discussed herein was selected because it was some of the most recent using the most current and reliable methods, the most relevant and the most frequently cited across numerous articles. As such, these studies and the researchers behind them appear to be forming the modern foundation for understanding and treatment of procrastination. And while most literature reviews would not necessarily concern themselves with research methods, the fact that results from these research studies form the cutting edge of our understanding of how procrastination occurs supported their inclusion in this paper.

Ferrari, Mason and Hammer (2006) utilized 120 college students (71 women, 47 men, 2 non-disclosed; M age 20.5 years old) from a Midwestern university who participated in order to get psychology course credit. The study used Lay's (1986) 20-item, 5-point ( 1 = not true of me, 5 = very true of me) General Procrastination Scale (GP). This scale examines behavioral procrastination tendencies, that is, delays in the start of completion of everyday tasks (Ferrari, Mason and Hammer, 2006). This scale has acceptable temporal stability (retest $r$ greater than or equal to 0.60 ) and internal consistency (present sample alpha $=0.89$; $\mathrm{M}$ score $=66.32, \mathrm{SD}=11.11$ ), as well as construct and predictive validities for use as a research inventory (see Ferrari et al., 1995, as cited in Ferrari, Mason and Hammer, 2006). Participants were then asked to give responses regarding tasks with a past deadline, a present deadline or a future deadline. Perceptions of both delayed and non-delayed tasks served as the dependent variables in the study (Ferrari, Mason and Hammer, 2006).

This study found that for delayed past tasks, procrastinators were more likely to see the task as difficult, not enjoyable and as a task that required a larger amount of effort. As well, procrastinators were more likely to state that they lacked clarity in how to complete the task. However, if they had completed the task, procrastinators believed it would have had a positive personal impact. Procrastination also predicted lower perceived enjoyment for delayed-current tasks (Ferrari, Mason and Hammer, 2006).

Ferrari, Mason and Hammer (2006) noted limitations to their present research, largely based on the fact that the research was exploratory in nature. They urge future research to confirm and expand their findings with these five variables - task difficulty, effort, enjoyableness, impact, and clarity - as affected by levels in procrastination.

And they suggest future research should consider populations other than college students such as working adults with real-world deadlines. Nevertheless, they feel the present study adds to the current body of personality research by demonstrating that individual differences in procrastination may be related to how people cognitively reframe their perceptions of task postponements as related to task deadlines (Ferrari, Mason and Hammer, 2006).

DeWitte and Schouwenburg (2002) studied college students as well. They worked with 147 freshmen (130 women (88.4\%), 17 men) enrolled in educational sciences at the University of Leuven (Belgium, Dutch speaking part). Their ages ranged from 17 to 42 (M1/418.6, SD1/42.1). DeWitte and Schouwenburg used two different methodologies to test both hypotheses in two ways. In the first phase of this study, they used the recently 
developed [emphasis added] Impulsivity test of Whiteside and Lynam (UPPS, 2001). Starting from a broad battery of available impulsivity scales and some additional items that were lacking from extant literature [emphasis added], they constructed a questionnaire measuring all aspects of impulsivity that have been investigated in the past. Four distinct factors were extracted: (lack of) Perseverance, (lack of) Premeditation, Urgency and Sensation seeking. The second phase of testing involved measurement of intentions versus completed work in a subsample of the first study (DeWitte and Schouwenburg, 2002).

DeWitte and Schouwenburg found procrastinators to be highly motivated students who suffer from a serious problem: they are vulnerable to temptations (2002). In addition, their study showed "multiple evidence that trait procrastination is related to impulsivity" (DeWitte and Schouwenburg, p. 485).

Lay's instrument was found to be internally consistent with a Cronberg's alpha of .89. Other measures showed similar validity levels. So, in this case, the results of the study can be considered valid. However, as the researchers themselves counsel, more research needs to be done to confirm their results, as they relied upon the relatively new Impulsivity test from Whiteside and Lynam, and they again turned to the convenience sample of available college students as the basis of their research. As they further note, this does not make their research invalid, but more study could expand the findings to more generalized usage (DeWitte and Schouwenburg, 2002).

Schouwenburg and Lay (1995) conducted two related studies. In Study 1, they used 161 female and 117 male university students in the Netherlands. In Study 2, respondents were Canadian university students (271 female, 81 male). The first study utilized Lay's (1986) Procrastination Scale, the same one used by Ferrari, Mason and Hammer, to create a trait-based portrait of the procrastinator. Study 2 examined the generality of the Study 1 findings and tied the traits to the Big 5 Personality Factors (Openness, Conscientiousness, Extraversion, Agreeableness, and Neuroticism) (Schouwenburg and Lay (1995)). Validity of both studies was high, with Cronberg's alpha at .80 or above.

These two studies were conducted with different methodologies, in different languages and in different countries, yet produced what their authors called "a striking resemblance" in their main result. The overwhelming association was with (lack of) Conscientiousness. They believe this should zero in future research on this particular association, that explanations for procrastination must have lack of conscientiousness at their core. In addition, this research claims to have debunked the notion that procrastinators seek an emotional high from delay (Schouwenburg and Lay (1995)).

While the validity of the research is clearly stated, and research has demonstrated the connection between lack of conscientiousness and procrastination, it seems somewhat early to be closing the door on other contributors to procrastination, and to call for limit and narrowing of research into a problem that has only recently begun to be explored. Schouwenburg and Lay (1995) are to be commended for their efforts and enthusiasm, but their success appears to call for more research and verification, not less.

Finally, Ferrari, O'Callaghan and Newbegin (2005) studied chronic procrastination in adult samples from the United States (122 women, 85 men), the United Kingdom (143 women, 96 men) and Australia (124 women, 90 men). They relied on a convenience sample, and utilized Lay's (1986) General Procrastination scale once again. In addition, they completed the 15-item, self-report Adult Inventory of Procrastination (McCown and Johnson, 1989, as cited in Ferrari, O'Callaghan and Newbegin, 2005).

They report that participants completed reliable and valid self-report measures of arousal procrastination (delays motivated by a last minute thrill experience) and avoidant procrastination (delays related to fears of failure or success). Overall, when both types were separated statistically into "pure types" there were no significant differences across countries: $11.5 \%$ of adults self-identified as arousal procrastinators, and $9.9 \%$ of adults as avoidant procrastinators. Thus, their results indicate that chronic procrastination is prevalent among westernized, individualistic, English-speaking countries, though they believe further epidemiological cross-cultural studies are needed. They found virtually no variance between men and women.

The authors note the need for testing on a random sample of people, different socio-economic groups, varied metropolitan settings and sub-cultures in each country. Further, they note that their study was exploratory in nature, as there was no previous research investigating prevalence rates across nationalities; as a result, they did not even form a hypothesis for their study. Rather than invalidating the conclusions reached in this study, these limitations again emphasize the need for further formal research on what is clearly a global problem.

\section{Literature in Existence}

The available material regarding procrastination shows that much of the research is of very recent vintage. Knaus 
(2000) notes that "prior to 1979, procrastination received limited attention in the United States" (p.153). As late as 2005 , Ferrari, O'Callaghan and Newbegin wrote that "no systematic study has examined the global prevalence of chronic procrastination - the purpose delay in starting or completing tasks" $(2005$, p. 2).

Ferrari, individually and in affiliation with other researchers, has written extensively on the issue and conducted extensive original research. Schouwenburg and Lay have linked the Netherlands and the United States, respectively, in some of the few cross-cultural studies of procrastination, doing considerable well-received research on the matter. The massive meta-analysis by Steel (2007) proves that, once in a great while, Sisyphus does indeed get the rock to the top of the hill. That author drew upon 691 correlations to create his review of books, articles and 553 research studies on procrastination, creating arguably the most comprehensive look at the issue to date. The reader is referred to this article as perhaps the best source for both a broad and specific view of studies and writings on this issue.

Steel explains both the condition itself, and the somewhat foggy understanding of it, when he calls procrastination a "prevalent and pernicious form of self-regulatory failure that is not entirely understood" (Steel, 2007, p. 65). Schouwenburg and Lay have defined trait procrastination as the lack of conscientiousness, going so far as to declare that "all primary interpretations of the procrastinator should now fit within this framework" and that for any other explanatory concepts researchers wish to introduce in the future, "some element of lack of conscientiousness must be at its core." More succinctly, they define procrastination as "to put off acting on one's intentions" (Schouwenburg and Lay, 1995, p. 481). Bui (2007) says that chronic procrastination represents a significant impediment to productivity for a wide range of people (p. 197). Ferrari, O'Callaghan and Newbegin (2005) labeled it the "purposive delay in starting or completing tasks" (p. 2). And Kotler calls procrastination a "gap between intention and action" (2009, p. 99.)

Numerous studies support the notion that procrastination, far from being a laughing matter, is a destructive force in many lives. O'Brien (2002, as cited in Steel, 2007) found that more than $95 \%$ of procrastinators wish to reduce it. According to Ellis and Knaus (1977), 80-95\% of college students engage in procrastination, and almost $50 \%$ procrastinate consistently and problematically (Day, Mensink, \&O'Sullivan, 2000; Haycock, 1993; Micek, 1982; Onwuegbuzie, 2000a; Solomon \& Rothblum, 1984, all as cited in Steel, 2007). Procrastination appears to be fairly evenly distributed by gender. Gender differences related to the construct of self-control has found mixed results (Feingold, 1994, as cited in Steel, 2007). Steel's 2007 meta-analytic results show that, on balance, one could expect procrastination to be weakly associated with males.

The problem extends far beyond the college classroom: procrastination chronically affects $15-20 \%$ of adults (Harriott \& Ferrari, 1996; "Haven't Filed Yet," 2003, as cited in Steel, 2007). And it predicts decreased work (Ellis \& Knaus, in press; Robb, 1998, as cited in Knaus, 2000) and academic performance (Owens \& Newbegin, 1977, as cited in Knaus, 2000). Ferrari, O'Callaghan and Newbegin (2005) found that it is more likely to be reported by white collar as compared to blue collar workers (Hammer and Ferrari, 2002, as cited in Ferrari, O'Callaghan and Newbegin, 2005).

Evidence suggests there can be little argument that procrastination causes significant problems. Briody (1980) notes that people characterize it as harmful, bad and foolish (as cited in Steel, 2007). Knaus (1973) and Lay \& Schouwenburg (1993) found that procrastinators are more miserable in the long term. Procrastination exacts a financial toll, as shown in an H\&R Block survey reporting that procrastination costs people an average of $\$ 400$ due to rushing and consequent errors (Kaster, 2004, as cited in Steel, 2007). Many other fields echo these findings, including medicine (Bogg and Roberts, 2004) and economics (Aklerof, 1991; O’Donoghue and Rabin, 1999, both as cited in Steel, 2007).

As the research shows, procrastination exacts a toll on many fronts. But what is procrastination? When does it go beyond minor delay, or reasonable prioritization, and become problematic? Are there degrees of procrastination? Knaus (2000) calls procrastination at the extreme a "highly change-resistant, persistent, emotionally distressing, problem habit" (p. 153). Steel (2007) cites a study by Arvey, Rotundo, Johnson, and McGue (2003) that there appears to be a biological or genetic component to procrastination fueling behavioral actions. Ferrari, O'Callaghan and Newbegin distinguish between arousal procrastination (delays motivated by a last-minute thrill experience) and avoidant procrastination (delays related to fears of failure or success) $(2005$, p. 2). Andreou (2007) called it "necessarily irrational," as "one follows a course of action that one finds worse than another available alternative," resulting in "self-imposed frustration" (2007, p. 21). Schouwenburg and Lay defined procrastination simply as putting off acting on one's intentions that, in some individuals, may become a pervasive and habitual activity; in turn, this means procrastination may be viewed as a personality trait which generates repeated episodes of procrastinatory behavior (1995). 
Silver and Sabini observed that when one procrastinates, one is particularly likely to find oneself succumbing to "ephemeral pleasures," such as playing a quick game of pinball, or even seeking "ephemeral chores," such as organizing one's spice rack (1981, p. 214). Because ephemeral pleasures and chores are often either more immediately gratifying or at least less daunting or aversive than the tasks associated with one's more important goals, they can be quite tempting. And because they are often individually compatible with one's more important goals, they can be pursued for a while without significantly hindering one's chances of achieving one's goals. But because their individually negligible consumption of time adds up, consistently pursuing them can interfere with one's more important goals and land one in a situation that one finds unacceptable (Andreou, 2007).

Time is a factor in procrastination. Ferrari, Mason and Hammer (2006) note that procrastinators tend to not focus on future tasks, suggesting that procrastination involves some relation to time perceptions; the further off a task, the less likely is the procrastinator to attend to it. Procrastinators "tend not to focus on future tasks needing attention," delaying an intended target task while often engaging in an irrelevant task (Ferrari, Mason and Hammer, 2006, p. 29). DeWitte and Schouwenberg observed that "procrastinators may have troubles appreciating the consequences that present choices have for the viability of remote goals. That is, they may underestimate the relevance of the present efforts for their final success" (2002, p. 471).

Why do we procrastinate at all? Schouwenberg (1992) stated that procrastinating students generally endorse many reasons simultaneously. One popular belief is that people procrastinate out of a fear of failure. But a study by Schouwenberg (1992) concluded that "fear of failure as a reason for procrastination is either of only secondary importance or practically absent." A study by Haycock (1993, as cited in Steel, 2007) found that only $7 \%$ of people surveyed reported perfectionism as contributing to their procrastination, so that common belief is not supported by research. Sensation seeking, that is, waiting till the last minute because doing so is more exciting, may be more a planned strategy; this would make it a rational strategy (although one whose benefits may be found to have been bought with diminished performance and, ultimately, long-term regret) and as such, not part of the definition of procrastination as an irrational act, according to Steel (2007). In addition, evidence suggests a weak causal link, with the average correlation between procrastination and sensations seeking being only .17 (Steel, 2007). Further, Steel (2007) found that irrational beliefs may be the source of some procrastination, and that although "clinical work stresses that irrational beliefs are a major source of procrastination, results have been irregular and often weak" (p. 36).

\section{Discussion}

If those common beliefs are not the research-supported causes of procrastination, then what are the causes? And can anything be done to help those suffering from the effects of procrastination?

Numerous researchers found that two top-rated reasons for procrastinating were that the task was unpleasant, or that it was boring and uninteresting (Anderson, 2001; Briody, 1980; Froehlich, 1987; Haycock, 1993, as cited in Steel, 2007). In turn, this may explain why some people procrastinate more than others, as they simply find more of life's chores and duties aversive (Steel, 2007). A contributing factor as well is the nature of our hunter-gatherer brains: by their design, more interest is expressed in immediate tasks than abstract tasks in the future (Kotler, 2009; Gropel \& Piers, 2011). The further away a deadline, the less urgent completion of a task feels. Procrastinators tend not to have a future temporal orientation (Lasane \& Jones, 2000; Specter \& Ferrari, 2000, as cited in Steel, 2007). Low self-efficacy and low self-esteem are associated with procrastination (Steel, 2007). In addition, depression is a culprit, with tiredness being one of the top three reasons that students give for putting off work (Strongman \& Burt, 2000, as cited in Steel, 2007).

Procrastination can play out as impulsivity and distractibility as well. Impulsivity can be defined in part as the tendency to give in to temptation (Costa and McCrae, 1980, as cited in Schouwenburg and Lay, 1995) as well as feeling overwhelmed by desires and drives (Schouwenburg and Lay, 1995). Impulsivity played a "solid role in procrastination," producing an average correlation between procrastination and impulsiveness of .41, according to Steel (2007). (It should be noted, however, that DeWitte and Schouwenburg claim that "previous evidence on the role of impulsivity in procrastination is ambiguous" [2002, p. 29]). Procrastination is typically impulsive and unplanned (Quarton, 1992, as cited in Steel, 2007). Procrastinators tend to choose short-term benefits over long-term gains, reflecting a core component of poor self-regulation (Tice \& Baumeister, 1997, as cited in Steel, 2007). As for distractibility, this has been identified by Haycock (1993) as one of the top reasons for procrastination (as cited in Steel, 2007).

The root cause of the dependent variable, procrastination, has been shown to be partially genetic in some cases, yet has many environmental and attitudinal influences, or independent variables, as well. Far from inhibiting solutions to the problem, however, this wide array of causes in fact suggests multiple solutions to this problem. 
For example, hopeful news comes from Steel (2007), who states that "people should procrastinate less as they age and learn" and notes that "it is evident that people can learn to avoid procrastination. Ainslie (1992) and Baumeister et al. (1994) reviewed considerable research showing that people tend to procrastinate less with repeated practice (cited in Steel, 2007). O’Donoghue and Rabin (1999) concluded, "Many people who procrastinate only moderately do so not because of intrinsic self-control, but because they have developed schemes to overcome procrastination" (as cited in Steel, 2007, p. 71). So, it appears the problem of procrastination may lessen with age.

Steel (2007) endorses many tenets of the Temporal Motivation Theory (TMT) to deal with the issue of procrastination. This theory includes reducing delay by increasing the expectancy of success with a task; generally, this makes the task much less aversive. A counter-intuitive suggestion is to increase the difficulty of the task, as this should reduce boredom and increase satisfaction of doing something difficult, rather than easy. However, going too far the other way, and making a task seem impossible, can induce procrastination as well, thus Steel (2007, p. 82) notes that "tasks should be constructed to be challenging but still achievable." The difficulty with distorted or discounted temporal perception of distant goals being unimportant could be addressed by pairing a distant goal with an immediate one, thus indulging a short-term impulse while supporting a larger objective (Steel, 2007). Additionally, classical conditioning can build learned industriousness, as rewards are matched immediately to successes large and small (Steel, 2007).

Andreou (2007, p. 190) cites studies by Ariely and Wertenbroch (2002) that "established that procrastinators are willing to impose binding deadlines on themselves, even when failure to meet their self-imposed deadlines will result in significant penalties. As one would expect, these self-imposed deadlines, which have bite, effectively improve task performance." Steel (2007) observes that:

Procrastinators tend to be impulsive, distractible, and lacking in self-control; thus, they are very sensitive to delays. Given procrastination's association with distractibility and organization, two methods of reducing distractions can be immediately recommended: stimulus control and automaticity (p. 25).

Stimulus control can consist of something as simple as working in an office, where distractions are minimized, or turning off email while working on a project; indeed, some $90 \%$ of college computer users admit to using email to delay irrationally (Brackin, Ferguson, Skelly, \& Chambliss, 2000, as cited in Steel, 2007). Automaticity consists of minimizing the number of "choice points" a task requires; few choices mean fewer delays (Silver, 1974, as cited in Steel, 2007). Finally, increasing organization and setting goals have both been shown to reduce procrastination (Steel, 2007).

\section{Conclusion}

We appear to be on the frontier of procrastination, aware of its presence and its implications, but not whole-heartedly embracing it as, say, a DSM-worthy malady. Nor do we have it in our sights enough, as clinicians or researchers, to drive a stake through its heart. Prevention, treatment or elimination of problematic procrastination is no easy task, and no simple solutions are at hand. The problem manifests in similar ways, yet finds different motivations in different individuals. Thus, as Andreou (2007, p. 190) observes, "understanding procrastination (including both its voluntary and self-defeating aspects) is a philosophically challenging task."

The most research work has been done in academia, presumably because that is where researchers and numerous "willing" subjects, aka students, comingle. In addition, some research work has been done on the effects of procrastination in the workplace, but not nearly enough to be considered comprehensive. As for the effects and manifestations of procrastination in the personal, private or home life, not even Steel's magnum opus collection could unearth anything resembling a definitive study of procrastination in those settings.

Should more research be done? Judging by the psychological, economic and societal costs associated with procrastination, the answer must be a resounding "yes." There appears to be a snowball effect taking place, as disparate research efforts get linked up through review articles, solidifying a research-supported knowledge base, and in turn spurring more research. Further, as research supports the reality of procrastination, perhaps it will be give the significance with clinicians (and the distinction of a treatable malady within a future edition of the DSM) that this author believes it deserves, and that this review paper supports. As society becomes more complicated, faster paced and more filled with distractions, procrastination is sure to increase.

Procrastination has been with humankind for countless millennia; only now are we taking it seriously, and taking it on. Why the delay, one wonders?

Why else? Procrastination. 


\section{References}

Achor, S. (2010). The happiness advantage: The seven principles of positive psychology that fuel success and performance at work. New York: Crown Business.

Andreou, C. (2007). Understanding procrastination. Journal for the Theory of Social Behaviour, 37(2), 183-193. http://dx.doi.org/10.1111/j.1468-5914.2007.00331.x

Apple. (2005). Apple dictionary. Cupertino, CA: Apple Inc.

Bui, N. (2007). Effect of evaluation threat on procrastination behavior. Journal of Social Psychology, 147(3), 197-210. http://dx.doi.org/10.3200/SOCP.147.3.197-209

Dewitt, S., \& Schouwenburg, H. (2002). Procrastination, temptations, and incentives: the struggle between the present and the future in procrastinators and the punctual. European Journal of Personality, 16(6), 469-489. http://dx.doi.org/10.1002/per.461

Ferrari, J., Mason, C., \& Hammer, C. (2006). Procrastination as a predictor of task perceptions: Examining delayed and non-delayed tasks across varied deadlines. Individual Differences Research, 4(1), 28-36.

Ferrari, J., O'Callaghan, J., \& Newbegin, I. (2005). Prevalence of procrastination in the United States, United Kingdom, and Australia: Arousal and avoidance delays among adults. North American Journal of Psychology, $7(1), 2-6$.

Gropel, P., \& Piers S. (2011). A mega-trial investigation of goal setting, interest enhancement, and energy on procrastination. Personality and Individual Differences, 45(5), 406-411. http://dx.doi.org/10.1016/j.paid.2008.05.015

Knaus, W. (2000). Procrastination, blame, and change. Journal of Social Behavior \& Personality, 15(5), 153-166.

Kotler, S. (2009). Escape artists. Psychology Today, 42(5), 72-79.

Schouwenburg, H. (1992). Procrastinators and fear of failure: an exploration of reasons for procrastination. European Journal of Personality, 6(3), 225-236. http://dx.doi.org/10.1002/per.2410060305

Schouwenburg, H., \& Lay, C. (1995). Trait procrastination and the big-five factors of personality. Personality and Individual Differences, 18(4), 481-490. http://dx.doi.org/10.1016/0191-8869(94)00176-S

Shakespeare, W. (1997). Measure for measure. New York, NY: Washington Square Press.

Steel, P. (2007). The nature of procrastination. Psychological Bulletin, 133(1), 65-94. http://dx.doi.org/10.1037/0033-2909.133.1.65 